\title{
Water quality simulation modeling and uncertainty analysis for risk assessment and decision making
}

\author{
Kenneth H. Reckhow \\ Center for the Analysis and Prediction of River Basin Environmental Systems (CARES) \\ Nicholas School of the Environment and Earth Sciences \\ Duke University, Durham, NC 27708-0328, USA \\ (Received 20 August 1992; accepted 16 March 1993)
}

\begin{abstract}
The usefulness of water quality simulation models for environmental management is explored with a focus on prediction uncertainty. Ecological risk and environmental analysis often involve scientific assessments that are highly uncertain. Still, environmental management decisions are being made, often with the support of a mathematical simulation model. In the area of pollutant transport and fate in surface waters, few of the extant simulation models have been rigorously evaluated. Limited observational data and limited scientific knowledge are often incompatible with the highly-detailed model structures of the large pollutant transport and fate models. Two examples are presented to illustrate data and knowledge weaknesses that are likely to undermine these large models for decision support. An alternative to comprehensive structured simulation models is proposed as a flexible approach to introduce science into the environmental risk assessment and decision making process.
\end{abstract}

\section{INTRODUCTION}

The recent interest in ecological risk assessment in the United States, and in particular in the US Environmental Protection Agency (EPA), has led to the realization that scientific uncertainties must be given more attention than in current practice. Indeed, the USEPA has recently recommended that uncertainty analyses be a routine part of ecological risk assessments (Risk Assessment Forum, 1992). This includes risk assessments that are proposed to make use of detailed pollutant transport and fate models.

Certainly the new emphasis on uncertainty analysis in environmental assessments can be viewed as a positive development. In environmental decision making, there are compelling reasons to always conduct uncertainty analyses, to avoid the mistaken impression that assessments are precise and well-understood: 
1. Environmental managers need to know the expected uncertainty in the assessed response. This should be an a priori estimate so that managers or decision makers may opt for another (more precisely-assessed) endpoint, may consider the value of additional experimentation or monitoring, and/or may hedge decisions away from large losses.

2. EPA, Congress, the public, etc. need to know the extent of critical scientific uncertainties in environmental assessments, so that future research funding is obtained and properly focused.

Scientific uncertainty is present in all ecological simulation modeling and risk assessments. Uncertainty does not prevent management and decision making; rather, it provides a basis for selecting among alternative actions and for deciding if (and what) additional information (experimentation and/or observation) is needed (Morgan and Henrion, 1990; Reckhow, 1994). Uncertainty can be used in this way because the magnitude of the uncertainty provides a measure of value of information: the smaller the uncertainty, the more confident (and valuable) is the assessment. Not only does this hold for formal environmental assessments, but it also reflects our day-to-day reasoning and decision making. Perhaps the best example of this is the response to probabilistic forecasts of rain. Many of us adjust our outdoor plans on the basis of these probabilities. A forecast of "it will rain" or "it will not rain" is unsatisfactory; we like to know the odds and act according to our attitude toward risk. This same reasoning should apply to uncertainty in environmental assessment and decision making.

However, for these and other advantages of uncertainty analysis to be realized, the analysis of uncertainty must be complete. That is, all scientific uncertainties in an ecological risk assessment or in pollutant transport and fate modeling must be estimated and included in the analysis. Failure to be complete can result in decisions that are not only far from optimal but are far from satisfactory in outcome. This issue is of immediate concern because some of the models proposed for ecological risk assessments are large mechanistic models which have yet to be subjected to thorough uncertainty analyses. Further, the complexity and size of these models, coupled with the limited observational data bases that are usually available to support modeling efforts, may make it unlikely that a thorough uncertainty analysis will be conducted.

The objective of this paper is to examine the particular topic of surface water transport and fate modeling for environmental risk assessment and decision support under uncertainty. The examination continues from this introductory discussion of uncertainty analysis to a review of extant transport and fate models. Difficulties encountered in uncertainty analyses on existing models are discussed using examples from the literature. Two case studies are then presented illustrating knowledge and data weaknesses that 
undermine mechanistic transport and fate models. The paper concludes with a proposal for model selection in ecological risk assessments, in consideration of the need for uncertainty analysis.

\section{WATER QUALITY TRANSPORT AND FATE MODELS}

In the previous section, the meaning and importance of uncertainty in risk assessment and decision making are discussed. With that foundation, much of the remainder of this paper is devoted to an examination of surface water transport and fate models as methods for scientific analysis and prediction. Throughout the following sections, uncertainty in knowledge, data, and ultimately, predictions is considered as a key criterion for the usefulness of models as methods for assessment and decision making.

Water quality simulation models of pollutant transport and fate are usually categorized as either mechanistic or empirical. Mechanistic models (J0rgensen, 1980; Chapra and Reckhow, 1983; Thomann and Mueller, 1987; Ambrose et al., 1988) are based on the modeler's expression of theory as one or more mathematical equations describing transport and fate. The mechanistic model is intended to be a correct, although simplified, description of the system of interest. In contrast, an empirical water quality model is based on a statistical summary of observational data. The empirical modeler often uses the observational data to guide in the selection of the model specification and to provide estimates of parameters. In empirical models, correct theoretical description is of secondary concern to parameter estimation.

The conventional approach for the determination of parameters for mechanistic water quality simulation models is for the modeler to use his judgment in selecting parameters that are consistent with any available data as well as with tabulations of accepted coefficient values (e.g., Schnoor et al., 1987). Parameters are often selected in a sequential manner (perhaps based on a sensitivity analysis) and the model is finally judged adequate based on a visual comparison of predictions and observations. Formal mathematical optimization is not usually involved.

Parameter estimation in empirical water quality models has traditionally been undertaken using classical optimization methods such as maximum likelihood or least squares. Judgment is typically involved in the specification of the model but not in the actual estimation algorithm.

For the mechanistic models, the governing philosophy is that correct description of processes in the models enhances the prospect for successful application of the models to previously unmodeled systems and for extrapolation of the models beyond the bounds of previous application. In addition, greater mechanistic detail should increase the chance that the model 
describes features of the problem that are of direct management concern. In theory, this philosophy yields versatile models that may be applied to a range of problems without major adjustments.

Until recently, none of the mechanistic pollutant transport and fate models supported by the USEPA included uncertainty analysis. This changed with QUAL2E-UNCAS (Brown and Barnwell, 1987), which is a significant contribution by EPA both as simulation model with uncertainty analysis and as a technical guidance document on the importance and interpretation of uncertainties. QUAL2E-UNCAS allows the user to select either first-order error analysis or Monte Carlo simulation for error propagation. The current version of the procedure does not include covariance terms or model equation error; unfortunately, this can result in prediction error estimates that severely misrepresent overall uncertainty as noted by Di Toro and van Straten (1979).

One difficulty encountered by Brown and Barnwell in QUAL2E-UNCAS is the limited amount of evidence on parameter error terms reported in the literature. In addition, it is not uncommon that, for the waterbody being modeled, there is only a small amount of observational data available to aid parameter selection, and even fewer data are typically left for model testing and evaluation. As a consequence, opportunities to rigorously assess success of these models in prediction have been rare and not necessarily meaningful.

To date, limited survey and anecdotal evidence suggest the following conclusions concerning the value of large mechanistic transport and fate modeling for surface water quality management:

1. Scientists and engineers at EPA, and others in the model development community (primarily at universities and at research laboratories), believe that these models are useful for water quality management. Among the reasons to support this perspective are the numbers and affiliations of attendees at EPA shortcourses, and the continued federal financial support for these models.

2. Scientists and engineers at state and regional agencies (who are the most common users of water quality simulation models) use the large mechanistic models infrequently for water quality management planning, with the exception of QUAL2E. Most of the routine modeling work (e.g., wasteload allocation studies) undertaken by these individuals is done using relatively simple models. Among the reasons for lack of use are that dollars and staff time are insufficient to support this level of modeling effort and skepticism among this group of model users that the large mechanistic models are reliable.

3. Many managers and decision makers without technical training in water quality modeling tend at first to favor use of a large mechanistic model 
for management decisions in the belief that it will yield more accurate, detailed predictions. Cost of application and difficulty in understanding the model and results appear to have ultimately led to a mixed view among members of this group.

4. Successful case studies, in which a mechanistic surface water quality model other than QUAL2E has aided decision making (and yielded predictions that have been shown to be fairly accurate) appear to be rare.

\section{HOW "GOOD" ARE EXTANT WATER QUALITY MODELS?}

There appear to be few essentially complete error analyses for predictive applications of surface water quality transport and fate models. The requirements for completeness adopted here are:

1. an estimate of error is chosen for all uncertain model terms, including inputs, initial conditions, and parameters, and covariances are included where appropriate;

2. an estimate of model equation error is determined. These error components are then combined using an error propagation method such as Monte Carlo simulation or first-order error analysis. An alternative approach involves:

3. model predictions compared to observations in a predictive scenario. For this approach to be acceptable, the model must be calibrated on a data set that is different from the data used for the predictive goodness-of-fit assessment. Being "different" does not mean simply different years of observation, as it is possible that essentially identical conditions will be observed each year. Rather, it means that the forcing functions and/or response are/ is different, and the degree of difference is assessed statistically and reported along with the goodness-of-fit results.

To make the error analysis feasible, minor error terms or errors that are found to have a negligible effect on predictions (perhaps due to insensitivity) can be ignored. In addition, error distribution shape may be difficult to characterize with confidence.

As noted above, few models come close to meeting these requirements. Among empirical models, only the steady-state nutrient input-output models have undergone reasonably thorough error analyses. For example, Reckhow and Chapra (1979) and Reckhow et al. (1992) report prediction error (based on leave-one-out cross-validation) of approximately 30\%-40\% for cross-sectional models that predict average growing season total phosphorus or total nitrogen concentration based on measured annual loading, Prediction errors are apt to be higher for applications based on estimated or predicted loading. Prediction error will be higher still when these simple 
models are linked to statistical models to predict chlorophyll $a$ or Secchi disk transparency.

Most error analyses conducted on mechanistic water quality models have also focused on eutrophication. For example, Scavia et al. (1981) applied error propagation methods to model several trophic state variables in Saginaw Bay, Lake Huron. In the uncertainty analysis, they included error terms for: (1) seven of the 22 parameters, (2) all of the eight initial conditions, (3) all of the five nutrient loads, and (4) the mixing parameter at the boundary between inner and outer Saginaw Bay segments. The remaining 15 (of 22) parameters and the model equations were assumed known without error. Results of this analysis indicate that, for the eight state variables (phytoplankton, herbivores, organic-N, ammonia, nitrate-nitrite, organic-P, $\mathrm{PO}_{4}$, and carnivores), the summer spatially-averaged daily coefficients of variation (CV) range from $33 \%$ (for organic-N) to $407 \%$ (for nitrate-nitrite), with maximum CVs of $148 \%$ to $772 \%$.

Scavia et al. did not include covariances in their error propagation study. In perhaps the most thorough study of parameter error involving covariances, Di Toro and van Straten (1979), and van Straten (1983), used maximum likelihood to determine point estimates and covariances for parameters in a seasonal phytoplankton model for Lake Ontario. Among the areas of investigation in these studies were the effect of unobserved state variables (no data on selected variables) on parameter errors, the importance of parameter covariance terms, and the consequences of fixing selected parameters at constant values.

Of particular note, Di Toro and van Straten found that prediction error decreased substantially when parameter covariances were included in error propagation, underscoring the importance of including covariance terms in error analyses. This result occurred because, while individual parameters might be highly uncertain, specific pairs of parameters (e.g., the halfsaturation constant and the maximum growth rate in the Michaelis-Menten model) may vary in a predictable way (expressed through covariance), and thus may be collectively less uncertain. For the error propagation study for the six nutrient and phytoplankton state variables, $\mathrm{Di}$ Toro and van Straten found the prediction coefficient of variation to range from $8 \%$ (for nitrate$\mathrm{N}$ ) to $390 \%$ (for ammonia-N), with half of the values falling between $44 \%$ and $91 \%$. Zooplankton prediction errors tended to be much higher.

In a study of uncertainty in modeling transport and fate for synthetic organics, Halfon (1984) used a mechanistic model to predict mirex behavior in Lake Ontario. Halfon noted the inadequacy of existing data to specify certain model functions and estimate particular model terms, and he attempted to assess the sensitivity of the model to uncertain parameters and look at prediction error. This analysis was done: (1) with the assump- 
tion that most parameters were known to within $\pm 20 \%$, (2) without a term for model error (Halfon did identify problems with the model), and (3) with no measurements of mirex loading (loadings were "back-calculated"). Halfon set the $\pm 20 \%$ parameter error level based on his sense of the literature, and used it as the full range (max-min) on the uncertainty in the parameters. This seems low (given natural variability, measurement error, and bias due to model mis-specification) for many of the model terms, particularly when used as an estimate of range. Unfortunately, Halfon's analysis and thoughtful discussion are constrained by data and model limitations, many of which he identifies.

Additional results presented by Beck (1987) in his thoughtful and thorough review paper indicate that the error levels cited in these studies are typical of those reported elsewhere. He concludes "Most of the evidence suggests that the current models of water quality, in particular, the larger models, are easily capable of generating predictions to which little confidence would be attached." Yet despite this view, there appears to be the belief among many modelers that since the world is complicated, so too must be our simulation models if they are to be accurate.

In a previous section, models were characterized as either mechanistic or empirical. In truth, simulation models exist on a continuum on a mechanistic (or empirical) axis, and the degree of mechanism attributed to a given model is apt to vary among individuals and among disciplines. For example, among water quality simulation modelers, WASP4 (Ambrose et al., 1988) is universally considered to be a mechanistic model. However, environmental chemists and aquatic biologists are apt to view the mathematics for the individual mechanisms as simplifications of the actual processes, and often as empirical simplifications, particularly when first-order reactions are employed. This does not mean that the simplifications are inappropriate. Rather, it means that "correct process description" should not be cited as the basis for selection of a "mechanistic" model.

Beck's conclusions concerning the problems with prediction error magnitude for the larger models raises interesting questions. Given current knowledge and available data, at what point does the level of detail in the model exceed the modeler's ability to produce an acceptable model? Can we model the transport and fate of every organism in a waterbody? Can we model the transport and fate of each species of phytoplankton in a waterbody? Can we model the transport and fate of major phytoplankton groups (e.g., greens, blue-greens, diatoms) in a waterbody? Can we model the transport and fate of overall phytoplankton biomass in a waterbody? A similar series of questions could be posed about space/time detail in a simulation model.

An interesting analogy to this is found in physics in the area of statistical 
mechanics. Physicists have recognized that, although they have an understanding of the theory and general agreement on the equations of motion. the movement of molecules or particles in gases or fluids is most effectively modeled by describing aggregate behavior, not by modeling individual molecules. Probability density functions are therefore used to define average response and variability in that response. Reif (1965) makes the key point quite effectively in the introductory chapter of his text on statistical and thermal physics:

'This book will be devoted to a discussion of systems consisting of very many particles... For, although it might be possible to write down the equations of motion for any one of these systems, the complexity of a system containing many particles is so great that it may make the task of deducing any useful consequences or predictions almost hopeless. The difficulties involved are not just questions of quantitative detail which can be solved by the brute force application of bigger and better computers. Indeed, even if the interactions between individual particles are rather simple, the shear complexity arising from the interaction of a large number of them can often give rise to quite unexpected qualitative features in the behavior of a system. It may require very deep analysis to predict the occurrence of these features from a knowledge of the individual particles... The task of understanding systems of many particles is thus far from trivial, even when the interactions between individual atoms are well known. Nor is the problem just one of carrying out complicated computations... When the systems under consideration are not too complex and when the desired level of description is not too detailed, considerable progress can indeed be achieved by relatively simple methods of analysis.'

This analogy is not intended as a recommendation that only highly aggregated, statistical behavior is the appropriate level of detail for water quality models. Rather, it is intended to support a call for thinking about the level of detail in a model as a compromise between what is desirable (what would we like to know?) and what is feasible (what is the best we can do, given current knowledge and available data?). This thought is addressed again below.

\section{PARAMETER DETERMINATION IN MECHANISTIC MODELS}

Mechanistic surface water quality models have been developed by scientists and engineers as mathematical descriptions of hydrologic and ecologic processes. Mechanistic modelers have tended to concentrate on the mathematical expression of theory, probably as a consequence of: (1) scientific interest and challenge, (2) a belief that the theory was reasonably well-understood and that this understanding could be expressed mathematically, 
(3) limited available data to fit and evaluate models, and (4) limited resources to collect additional data. For these reasons, model coefficients and reaction rates in the models generally characterize actual processes and are not (at least initially) intended to be empirically-fitted constants.

Since the parameters of mechanistic models are intended to describe real processes, it may be assumed that an experimental study of a particular process can yield a parameter estimate that can be directly inserted into the model. In some cases, it is acknowledged that the rate or coefficient in the model is affected by other conditions in the waterbody (e.g., turbulence), and thus adjustments must be made to the experimentally-based value. However, if the model truly is a complete mechanistic description of the system of interest, then adjustment should be unnecessary. This view is reflected in Halfon's (1984) statement concerning EXAMS (Burns and Cline, 1985): "Since the EXAMS model is based on documented formulas, it does not require calibration."

However, given the relative simplicity of all simulation models in comparison to the complexity of nature, it seems reasonable to question the legitimacy of any "mechanistic" mathematical description of surface water quality. Further, given data limitations and scientific knowledge limitations, it seems reasonable to question even the goal to strive for a model that need not be calibrated. The correctness of model structure, the knowledge of the model user, and the availability of experimental and observational evidence all influence parameter choice for mechanistic models. Unfortunately, too often knowledge and data are extremely limited, making choice of parameters and choice of important model processes guesswork to a distressingly large degree. The examples presented in the next two sections are not re-assuring with respect to these two issues: (1) scientific support for the selection of model parameters, and (2) scientific support for the specification of appropriate model functional relationships.

\section{AN EXAMPLE OF PARAMETER SELECTION APPROACHES: PHYTOPLANKTON SETTLING VELOCITY}

One of the basic parameters in an aquatic ecosystem model is phytoplankton settling. An early example of its use is in the model proposed by Chen and Orlob (1972):

$$
\frac{d V C_{1}}{d t}=Q C_{i n}+E A \frac{d C_{1}}{d t}-Q C_{1}+\left(\mu_{1}-R_{1}-s_{1}-M_{1}\right) V C_{1}-\mu_{2} V C_{2} F_{2,1}
$$

where:

$V=$ segment volume $\left(\mathrm{m}^{3}\right)$

$\mathrm{C}_{1}=$ phytoplankton concentration $\left(\mathrm{g} / \mathrm{m}^{3}\right)$ 


$$
\begin{aligned}
& Q=\text { flow volume }\left(\mathrm{m}^{3} / \mathrm{t}\right) \\
& E=\text { diffusion coefficient }\left(\mathrm{m}^{2} / \mathrm{t}\right) \\
& A=\text { segment surface/bottom area }\left(\mathrm{m}^{2}\right) \\
& \mu_{1}=\text { phytoplankton growth rate }\left(\mathrm{t}^{-1}\right) \\
& R_{1}=\text { phytoplankton respiration rate }\left(\mathrm{t}^{-1}\right) \\
& \mathrm{S}_{1}=\text { phytoplankton settling rate }\left(\mathrm{t}^{-1}\right) \\
& M_{1}=\text { phytoplankton mortality rate }\left(\mathrm{t}^{-1}\right) \\
& \mu_{2}=\text { zooplankton growth rate }\left(\mathrm{t}^{-1}\right) \\
& C_{2}=\text { zooplankton concentration }\left(\mathrm{g} / \mathrm{m}^{3}\right) \\
& F_{2, I}=\text { fractional feeding preference }
\end{aligned}
$$

Other examples are quite similar; a common alternative approach is that phytoplankton settling is sometimes treated as a velocity $\left(v_{l}\right)$ term with an areal loss (see Chapra and Reckhow, 1983, Chapter 14):

$$
\text { phytoplankton settling (mass/time) }=v_{l} A C_{l} \text {. }
$$

To understand some of the problems with the current approach for parameter determination in mechanistic surface water quality models, it is useful to examine this process further. For that purpose, "phytoplankton settling velocity" provides a good example. Phytoplankton, or algae, are important in aquatic ecosystems, and thus one or more phytoplankton compartments are found in most mechanistic surface water quality models concerned with nutrient enrichment. Phytoplankton settling is one of the key mechanisms for removal of phytoplankton from the water column.

Stoke's law provides the starting point for the mathematical characterization of phytoplankton settling. Few models, however, employ Stoke's law; instead a simple constant settling velocity (in units of length/time) expression is commonly used. To apply a model with this settling velocity term, a modeler must either measure phytoplankton settling directly, or select a representative value from an other study. Since field measurement of phytoplankton settling is a difficult task, use of literature-tabulated values is standard practice.

Probably the most thorough listing of suggested values for phytoplankton settling velocity is Bowie et al. (1985), which presents a two-page table of reported values, by algal type (see Table 1). Bowie et al. note that under quiescent conditions in the laboratory, phytoplankton settling is a function of algal cell radius, shape, density, and special cell features such as gas vacuoles and gelatinous sheaths. For natural water bodies, water turbulence can be quite important. In two- or three-dimensional models with hydrodynamic simulation, turbulence is accounted for in the model equations; in zero- or one-dimensional models, the effect of turbulence on phytoplankton settling must usually be incorporated into the choice of settling velocity. 
TABLE 1

Phytoplankton settling velocities (Bowie et al., 1985) ${ }^{\text {a }}$

\begin{tabular}{|c|c|c|c|}
\hline Algal type & $\begin{array}{l}\text { Settling velocity } \\
(\mathrm{m} / \text { day })\end{array}$ & Algal type & $\begin{array}{l}\text { Settling velocity } \\
(\mathrm{m} / \text { day })\end{array}$ \\
\hline \multirow[t]{11}{*}{ Total phytoplankton } & $0.05-0.5$ & Green algae & $0.05-0.19$ \\
\hline & $0.05-0.2$ & & $0.05-0.4$ \\
\hline & $0.02-0.05$ & & 0.02 \\
\hline & 0.4 & & 0.8 \\
\hline & $0.03-0.05$ & & $0.1-0.25$ \\
\hline & 0.05 & & 0.3 \\
\hline & $0.2-0.25$ & & $0.08-0.18$ \\
\hline & $0.04-0.6$ & & $0.27-0.89$ \\
\hline & $0.01-4.0$ & Blue-green algae & $0.05-0.15$ \\
\hline & $0-2.0$ & & 0 \\
\hline & $0-30$ & & 0.2 \\
\hline \multirow[t]{12}{*}{ Diatoms } & $0.05-0.4$ & & 0.1 \\
\hline & $0.1-0.2$ & & $0.08-0.2$ \\
\hline & $0.1-0.25$ & & $0.10-0.11$ \\
\hline & $0.03-0.05$ & Flagellates & 0.5 \\
\hline & $0.3-0.5$ & & 0.05 \\
\hline & 2.5 & & $0.09-0.2$ \\
\hline & $0.02-14.7$ & & $0.07-0.39$ \\
\hline & $0.08-17.1$ & Dinoflagellates & 8 \\
\hline & & & $2.8-6.0$ \\
\hline & & Chrysophytes & 0.5 \\
\hline & & Coccolithophores & $0.25-13.6$ \\
\hline & & & $0.3-1.5$ \\
\hline
\end{tabular}

${ }^{\mathrm{a}}$ See Bowie et al. (1985) for original references for reported settling velocities.

That information is typically the extent of technical guidance employed by modelers when selecting this parameter using a reference like Table 1 from Bowie et al. (1985). The range of options in Table 1 is substantial, even within a single category (e.g., diatoms) for algal type. The algal cell size, shape, and other features mentioned in the previous paragraph can vary from species to species within a single type category, so this may be responsible for some of the variability in Table 1. However, even if the modeler who must choose a point estimate has data that identify dominant species in a water body at a particular time and location, dominance is apt to change with time and location. Further, models contain at most only a few distinct phytoplankton compartments, so a choice must still be made concerning species to be modeled and their characteristics.

Examination of the original references from which Table 1 was created does little to enlighten the parameter selection process. Most of the references summarized in Table 1 do not present observational studies on 
phytoplankton; rather, they are simulation model studies, and the value for phytoplankton settling velocity listed in Table 1 is the value chosen for the model. In some of the references checked, little or no basis was provided for the choice. When a rationale for choice was given, it was usually to adopt or adjust the few values presented in the literature from experimental studies, or to adopt a value from another modeling study. In one way or another, it appears that virtually all of the values presented in Table 1 have some dependency on the early experimental work of Smayda and Boleyn (1965) and other work by Smayda.

Unfortunately, evaluation studies of simulation models have provided little insight on good point estimates for this parameter. Observational data on surface water quality are almost always inadequate for testing functional relationships and assessing parameter choices. Typical observational data sets are noisy, with few measurements of each of only a few variables. In the case of phytoplankton settling velocity, observational data are apt to consist of phytoplankton cell densities at various dates, times, and areal locations, but probably not depths. Since phytoplankton are also removed from the water column through consumption by higher food chain organisms, the observational data do not permit separate identification of the removal mechanisms.

Given this situation, modelers have relied almost exclusively on the few experimental studies in the laboratory and their judgment concerning adjustments to these values. For one-dimensional models without explicit modeling of hydrodynamics, the chosen value may be as much as an order of magnitude higher than the laboratory values. Two- or three-dimensional models with hydrodynamics may incorporate the unadjusted laboratory value. After early modeling studies presented chosen values, these values were sometimes adopted in subsequent studies without comment (in effect, "default" values were identified). Thus, there is probably much less information in the columns of Table 1 than implied by the number of values reported.

In summary, the choices for phytoplankton settling velocity appear to be based on ad hoc adjustments to a few values measured under controlled conditions. There is virtually no field confirmation of choices made for parameters individually (as opposed to collectively). This situation is fairly typical of the state-of-the-art in surface water quality simulation modeling.

\section{FROM SCIENTIFIC KNOWLEDGE, EXPERIMENTATION, AND OBSERVATION TO MODEL SPECIFICATION: BIODEGRADATION OF PCBS}

Several transformations may affect the concentration of synthetic organic chemicals (SOCs) in natural waterbodies; among them are: volatiliza- 
tion, adsorption and sedimentation, hydrolysis, photolysis, and biodegradation (Chapra and Reckhow, 1983). Biodegradation covers a number of distinct processes including mineralization, detoxication, and metabolism. Biodegradation by-products may be inorganic, or they may be organic compounds that are simpler or related to the original SOC.

In most natural water bodies, SOCs are at relatively low concentrations. Bacteria tend to exist at fairly high stable population levels, and they generally prefer other more abundant carbon sources over SOCs. As a consequence, the Michaelis-Menten relationship, which is often used to model uptake and growth, is frequently simplified to a first-order expression when applied to the biodegradation of SOCs:

biodegradation loss (mass/time) $=k_{b} V c$,

where:

$$
\begin{aligned}
& k_{b}=\text { first-order degradation rate constant }\left(\mathrm{t}^{-1}\right) \\
& V=\text { system volume }\left(\mathrm{m}^{3}\right) \\
& c=\text { total contaminant concentration }\left(\mathrm{g} / \mathrm{m}^{3}\right)
\end{aligned}
$$

While it has been shown that most SOCs (like PCBs; polychlorinated biphenyls) are biodegradable under controlled conditions (e.g., in laboratory experiments), there is often a great deal of uncertainty concerning the magnitude, and even the existence, of biodegradation occurring naturally in waterbodies. As Madsen (1991) notes, "In situ biodegradation of organic compounds is very difficult to prove." For example, as Chapra and Reckhow (1983) observe, "many laboratory degradation studies are performed using microbial populations that have been acclimated to a particular organic compound. Whether such species would tend to behave similarly in the lake environment is questionable and makes it difficult to apply some of the laboratory work to natural situations." Unfortunately, Madsen (1991) concludes, "The problems of extrapolating from laboratory data to the field have never been solved despite decades of debate." Even with these difficulties, biodegradation is frequently included as a transformation process when models such as WASP4 are used for SOC transport and fate.

In a study of the scientific basis for parameter determination in simulation models, Wolpert et al. (1993) looked at biodegradation and other processes affecting PCBs in surface waterbodies. Their review of the mechanistic modeling literature indicated that for the most part, even in modeling studies with the most thorough databases, the source for the biodegradation rate constant either was not specified or was based on a synthesis of literature values. In other words, there did not appear to be in situ evidence for a particular biodegradation rate.

In their own modeling work on PCB transport and fate in the upper Hudson River (NY), Wolpert et al. (1993) stated "It is believed that there 
is some biodegradation of PCBs in the sediment [of the Hudson River], but even that is uncertain." The biodegradation rate depends on many factors including temperature, dissolved oxygen, nutrients such as nitrogen and phosphorus, and the mix and concentration of micro-organisms. Biodegradation may be either aerobic or anaerobic; if biodegradation occurs naturally, it seems plausible that aerobic processes could dominate in the water column and anaerobic processes could dominate in the sediments.

One problem that confronted Wolpert et al. (1993) in their modeling study is that PCBs exist not as a single compound, but as 209 congeners. These congeners are a function of the number and placement of chlorine atoms bonded to the ten carbon atoms in all PCBs. The congeners not only vary with chlorine, but they also vary by molecular weight (from 189 to 499 $\mathrm{g} / \mathrm{mol}$ ) and by chemical/physical behavior. Thus, in effect, PCB transport and fate prediction involves the modeling of 209 compounds. Unfortunately, as Wolpert et al. note, it is difficult to distinguish among congeners in natural water bodies using conventional monitoring techniques.

The literature on biodegradation rates illustrates one of the problems with the congeners. Bedard et al. (1986) measured aerobic biodegradation rate for PCBs in the laboratory. Over a 72-h period, they measured degradation rates varying from less than $20 \%$ to greater than $90 \%$, depending on congener. As a consequence, the effective first-order aerobic degradation rate for these experiments ranged from less than $0.86 \cdot 10^{-6} \mathrm{~s}^{-1}$ to greater than $17.7 \cdot 10^{-6} \mathrm{~s}^{-1}$. The situation is similar for anaerobic degradation. Under experimentally-controlled conditions, Rhee et al. (1979) found anaerobic degradation of PCB congeners from Hudson River sediment to range from $8 \%$ to $63 \%$ over a 7 -month period. This translates to first-order anaerobic biodegradation rates between $4.5 \cdot 10^{-9} \mathrm{~s}^{-1}$ and $54.0 \cdot 10^{-9} \mathrm{~s}^{-1}$, depending upon congener.

Even for a single congener, the measured biodegradation rates can vary. For two different congener mixtures, Bedard et al. (1986) measured PCB congener concentrations after exposure to bacteria. For a single congener (labeled 4, 4') common to both mixtures, the apparent degradation rate differed. As Wolpert et al. (1993) inquire, "Should this difference be attributed to:

- Measurement error?

- Different biodegradation rates, depending on the mixture of congeners present and their relative abundance?

- Biotransformation of one congener into another (with fewer chlorine atoms) through dechlorination, so that the reported differences are only the net changes in each congener?"

Focusing on the implications for modeling PCB transport and fate in the Hudson River, Wolpert et al. (1993) continue, "If the rates do depend on 
the mixture of PCBs present, it is hard to see how to use the reported rates to predict biodegradation rates in the Hudson River bed with its unknown mixture of PCB congeners unless each congener is modeled separately and it is known how different ratios of congener concentrations affect the biodegradation rates. Clearly, congener-specific degradation rates for all possible mixtures are unavailable. Our ability to model individual congeners is also limited by uncertainty about the initial congener composition of the river loadings and of the observed water column and sediment samples. Other reasons for suspecting the laboratory data may not reflect field conditions accurately include:

- It is likely that laboratory growing conditions are more conducive to bacterial populations than are river conditions. Populations of PCB-degrading bacteria could well be orders of magnitude smaller in the river than in the laboratory;

- The experiments do not include enough measurements over time to verify a stationary biodegradation rate constant, as assumed in [the] model;

- Environmental conditions in the sediment must be expected to differ from those in the laboratory - for example, the ratio of carbon dioxide to hydrogen is a particularly sensitive environmental parameter for bacteria and can vary from place to place in the sediment."

Initial reaction to the uncertainty regarding in situ biodegradation of PCBs is apt to be one of concern. This may be followed quickly by the observation that, in fact, PCBs are 209 compounds; thus, why expect that a single mechanistic model will be adequate for transport and fate? Perhaps so, yet:

- PCBs are among the most studied SOCs in the environment; thus, scientific knowledge, experimental evidence, and observational data should provide a "best case" for the state-of-the-art in SOC transport and fate modeling,

- Decisions (including the decision to do nothing) are being made concerning PCBs in the aquatic environment, and models should be considered for decision support.

Certainly, we must have an understanding of cause-effect relationships to make reliable predictions of pollutant transport and fate. Predictions based on statistical relationships alone may work for interpolation in well-studied situations (e.g., aggregate response for lake eutrophication), but they may fail to account for important mechanisms in new situations. Yet, uncertainties in the mechanistic transport and fate models should cause us to search for other ways to provide predictive scientific analyses for decision support. A suggested alternative is presented below. 
7. A PROPOSAL FOR MODEL SELECTION IN DECISION SUPPORT FOR RISK ASSESSMENT

As noted, prediction uncertainty is apt to be quite large for mechanistic transport and fate models like WASP4 and EXAMS. Among the reasons why there continues to be support for large water quality simulation models, even in a situation of apparently great uncertainty, are: (1) the belief that correct mathematical descriptions allow extrapolation to other unmodeled systems, and (2) the desire to fully capture within the model a complete description of a system from management controls to meaningful system responses. This second justification for large models presumably reflects a view that a decision support system should be based on an automated analysis that minimizes human intervention.

An alternative perspective may be found in the medical diagnosis area. Decision support may take the form of an expert system that receives information on symptoms and identifies likely causes. There is no attempt to replace the physician; rather, the expert system provides information to the physician to improve decision making. The physician interprets the evidence, including the output from the expert system, and makes a judgment and recommendation.

In surface water quality management, the experienced subject matter scientist may fail to identify patterns in multivariate data that a simulation model or data analysis may easily interpret. However, the scientist has experiences to provide insight and to interpret information in a more flexible way than does the logical but structured simulation model. When the subject matter scientist is aided by a simple expert system to help reveal patterns, the result could be both an ability to discover patterns and an effectiveness in interpreting their consequences. This improved interpretation may refer both to prediction of system response and to assessment of the nature and value of sample information to aid decision making.

Thus, one option for the design of a decision support system in environmental management is to specify the nature and scale of information to be presented to a subject area scientist, who then interprets the information for decision makers. Given the current state-of-the-art in transport and fate modeling, is decision making better served by large, detailed simulation models that describe a system from inputs to meaningful effects, even if the prediction uncertainty is extremely large? Or, will better decisions result if simple models are used to provide predictions of aggregate behavior for a subject matter scientist to interpret detailed response for decision makers?

The roles of: (1) the expert system for decision support, (2) the subject matter scientist, and (3) the decision maker may be guided by general criteria, and by certain specific criteria that may differ among subject areas. 
Ideally, as described above, there is interaction between the decision maker and the subject matter scientist in defining the scope of the analysis. Any simulation model that provides decision support must have uncertainties of acceptable magnitude; in some subject areas this may require simple aggregate-behavior models, while in other areas complex models may be useful.

The actual choice of simulation models used within this proposed decision support system should be based on a compromise between feasibility and desirability. To see how this might occur, consider the following hypothetical example as a way to interactively arrive at model choice:

Initially, the "environmental managers" should identify desirable objectives and attributes ("endpoints" or "measures of effectiveness"). The attributes represent quantities that are measurable (predictable) and meaningful to the issue of concern; for eutrophication, floating mats of algae (probably not chlorophyll $a$, unless a chla standard exists) is one possible example.

A possible next step is for the "environmental modelers" to identify the feasibility of a predictive scientific assessment of the proposed attributes, based on spatial, temporal, and ecological scales. This should be expressed in terms of scientific uncertainty (e.g., "We can predict abundance of the species with a standard error of $\pm 100 \% ")$.

Then, the environmental managers determine if this is acceptable, given the value function (e.g., losses or net benefits associated with management options); it is conceivable that the desired attribute has an undesirable level of predictive uncertainty (e.g., "We prefer abundance as an endpoint, but $\pm 100 \%$ is unacceptable. What alternatives can be predicted with greater precision?").

If the problem is then referred back to the environmental modelers, they may consider aggregation to reduce uncertainty (e.g., "We can predict presence/absence to $\pm 30 \%$; is that useful?"). This type of interaction is intended to lead to good compromise choices for the level of the analysis and the nature of the predicted response.

This interplay is critical, but for this interaction to be effective, we need both scientific uncertainty analyses and some notion of the value function. The environmental modeler should not solely determine the nature (cost of effort and level of detail) of the ecological assessment, yet the environmental manager should not dictate endpoints to the environmental modeler. There should be frequent interaction, with consideration of uncertainty and valuation, in order to balance what is desirable with what is feasible.

This decision theoretic approach helps us assess the value of new (sample) information; this, too, requires interaction between modelers and managers. For example, the need for additional experimentation or moni- 
toring is evaluated in terms of the value function (e.g., "Including the cost of experimentation or monitoring, is the expected utility higher or lower with additional information?"). In addition, a decision theoretic approach to environmental risk assessment illustrates the critical role of uncertainty analysis in the interaction between modelers and managers discussed above.

\section{A CONCLUDING THOUGHT}

No one can claim that scientific uncertainty is desirable; yet, no one should claim that scientific uncertainty is best hidden or ignored. Estimates of uncertainty in predictions are not unlike the point estimates of predicted response. Like the point predictions, the uncertainty estimates contain information that can improve risk assessment and decision making. The decision support system proposed above will not eliminate this uncertainty nor will it change the fact that, due to uncertainty, some decisions will yield consequences other than those anticipated. It will, however, allow risk assessors and decision makers to use the uncertainty to structure the analysis and present the scientific inferences in an appropriate way. In the long run, that should improve environmental management and decision making.

\section{ACKNOWLEDGMENTS}

The observations and ideas presented in this paper developed through many helpful discussions with Robert Wolpert, Tom Stockton, Laura Steinberg, and Katrina Smith. This work was supported under a grant from the National Science Foundation (SES-8921227).

\section{REFERENCES}

Ambrose, R.B., Jr., Wool, T.A., Connolly, J.P. and Schanz, R.W., 1988. WASP4, A Hydrodynamic and Water Quality Model - Model Theory, User's Manual, and Programmer's Guide. U.S. Environmental Protection Agency, EPA/600/3-87/039.

Beck, M.B., 1987. Water Quality Modeling: A Review of the Analysis of Uncertainty. Water Resour. Res., 23: 1393-1442.

Bedard, D.L., Unterman, R., Bopp, L.H., Brennan, M.J., Haberi, M.L. and Johnson, C., 1986. Rapid Assay for Screening and Characterizing Microorganisms for the Ability to Degrade Polychlorinated Biphenyls. Appl. Environ. Microbiol., 51: 761-768.

Bowie, G.L., Mills, W.B., Porcella, D.B., Campbell, C.L., Pagenkopf, J.R., Rupp, G.L., Johnson, K.M., Chan, P.W.H., Gherini, S.A. and Chamberlin. C.E., 1985. Rates. Constants, and Kinetics Formulations in Surface Water Quality Modeling. U.S. Environmental Protection Agency, EPA/600/3-85/040.

Brown, L.C. and Barnwell, T.O., Jr., 1987. The Enhanced Stream Water Quality Models QUAL2E and QUAL2E-UNCAS: Documentation and User Manual. U.S. Environmental Protection Agency, EPA/600/3-87/007.

Burns, L.A. and Cline, D.M., 1985. Exposure Analysis Modeling System, Reference Manual for EXAMS II.U.S. Environmental Protection Agency, EPA-600/3-85-038.

Chapra, S.C. and Reckhow, K.H., 1983. Engineering Approaches for Lake Management. Vol. II: Mechanistic Modeling. Butterworths, Boston, 492 pp.

Chen, C.W. and Orlob, G.T., 1972. Ecologic Simulation for Aquatic Environments. Office of Water Resources Research, US Dept. of Interior, Washington, DC. 
Di Toro, D.M. and van Straten, G., 1979. Uncertainty in the Parameters and Predictions of Phytoplankton Models. Working Paper WP-79-27, International Institute for Applied Systems Analysis, Laxenburg, Austria.

Halfon, E., 1984. Error analysis and simulation of mirex behaviour in Lake Ontario. Ecol. Modelling, 22: 213-252.

Jorgensen, S.E., 1980. Lake Management. Pergamon Press, Oxford, 167 pp.

Madsen, E.L., 1991. Determining in situ biodegradation - facts and challenges. Environ. Sci. Technol., 25: 1663-1673.

Morgan, M.G. and Henrion, M., 1990. Uncertainty. Cambridge University Press, Cambridge, 332 pp.

Reckhow, K.H., 1994. The importance of scientific uncertainty in decision making. Environmental Management. 18:161-166.

Reckhow, K.H. and Chapra, S.C., 1979. Error analysis for a phosphorus retention model. Water Resour. Res., 15: 1643-1646.

Reckhow, K.H., Coffey, S.C., Henning, M.H., Smith, K. and Banting, R., 1992. Eutromod: Technical Guidance and Spreadsheet Models for Nutrient Loading and Lake Eutrophication. Duke University School of the Environment, Durham, NC.

Reif, F., 1965. Fundamentals of Statistical and Thermal Physics. McGraw-Hill, New York, $651 \mathrm{pp}$.

Rhee, G.-Y., Bush, B., Brown, M.P., Kane, M. and Shane, L., 1989. Anaerobic biodegradation of polychlorinated biphenyls in Hudson River sediments and dredged sediments in clay encapsulation. Water Res., 23: 957-964.

Risk Assessment Forum, 1992. Framework for Ecological Risk Assessment. U.S. Environmental Protection Agency, EPA/630/R-92/001.

Scavia, D., Canale, R.P., Powers, W.F. and Moody, J.L., 1981. Variance Estimates for a Dynamic Eutrophication Model of Saginaw Bay, Lake Huron. Water Resour. Res., 17:1115-1124.

Schnoor, J.L., Sato, C., McKechnie, D. and Sahoo, D., 1987. Processes, Coefficients, and Models for Simulating Toxic Organics and Heavy metals in Surface Waters. U.S. Environmental Protection Agency, EPA/600/3-87/015.

Smayda, T.I. and Boleyn, B.J., 1965. Experimental observations on the floatation of marine diatoms. Part I: Thalassiosira naria, T. rotula and Nitzschia seriata. Limnol. Oceanogr., 10:499-510.

Thomann, R.V. and Mueller, J.A., 1987. Principles of Surface Water Quality Modeling and Control. Harper \& Row, New York, 644 pp.

van Straten, G., 1983. Maximum likelihood estimation of parameters and uncertainty in phytoplankton models. In: M.B. Beck and G. van Straten (Editors), Uncertainty and Forecasting of Water Quality. Springer Verlag, Berlin.

Wolpert, R.L., Steinberg, L.J. and Reckhow, K.H., 1993. Bayesian decision support using environmental transport and fate models. In: C. Gatsonis, J.S. Hodges, R.E. Kass and N.D. Singpurwalla (Editors), Case Studies in Bayesian Statistics. Springer, New York. 